Cahiers $d u$ MONDE RUSSE

\section{Cahiers du monde russe}

Russie - Empire russe - Union soviétique et États indépendants

$51 / 4 \mid 2010$

Sciences humaines et sociales en Russie à l'Âge d'argent

\title{
Moshe Gammer, ed., Ethno-Nationalism, Islam and the State in the Caucasus
}

\section{Stéphane A. Dudoignon}

\section{(2) OpenEdition}

\section{Journals}

Édition électronique

URL : https://journals.openedition.org/monderusse/7436

DOI : 10.4000/monderusse. 7436

ISSN : $1777-5388$

Éditeur

Éditions de l'EHESS

\section{Édition imprimée}

Date de publication : 25 novembre 2010

Pagination : 799-803

ISBN : 978-2-7132-2316-7

ISSN : $1252-6576$

Référence électronique

Stéphane A. Dudoignon, " Moshe Gammer, ed., Ethno-Nationalism, Islam and the State in the Caucasus ", Cahiers du monde russe [En ligne], 51/4 | 2010, mis en ligne le 09 décembre 2011, consulté le 03 septembre 2022. URL : http://journals.openedition.org/monderusse/7436 ; DOI https://doi.org/10.4000/monderusse.7436

Ce document a été généré automatiquement le 3 septembre 2022.

Tous droits réservés 


\title{
Moshe Gammer, ed., Ethno- Nationalism, Islam and the State in the Caucasus
}

\author{
Stéphane A. Dudoignon
}

\section{RÉFÉRENCE}

Moshe GAMMER, ed., Ethno-Nationalism, Islam and the State in the Caucasus. Londres - New York : Routledge, 2008, 233 p. (Central Asian Studies Series, 9)

1 Historien exceptionnellement prolifique du Caucase moderne et contemporain, Moshe Gammer nous avait déjà gratifiés, ces dernières années, de plusieurs volumes collectifs qui font date et balisent le renouveau actuel des études sur cette région de l'ancien monde soviétique, notamment par un effort de remise en contexte des évolutions de la pensée et de la pratique islamiques ${ }^{1}$. Du point de vue des études contemporaines, postsoviétiques en particulier, on retiendra, entre autres, plusieurs articles particulièrement innovants de sociologie politique ${ }^{2}$.

Mettant constamment en garde les lecteurs contre l'instrumentalisation politique faite dans l'ex-URSS de termes génériques empruntés à la littérature polémique (tels que " fondamentalisme », " wahhabisme », « extrémisme », etc.), M. Gammer propose une lecture historique du nationalisme et de l'islam dans le Caucase du Nord, au Daghestan en particulier, qui intègre les phénomènes économiques et sociaux les plus divers. Parmi ces phénomènes, on trouve : le rôle de la diversité ethnique et des rivalités entre groupes dominants (querelles entre Avars, Dargues et Kumyks pour le contrôle de l'appareil d'État et de la direction spirituelle musulmane du Daghestan); le poids des migrations et des déportations de la période soviétique (avec le retour impossible sur leurs terres des Aki Tchétchènes du Daghestan après leur réhabilitation); la continuité du personnel politique hérité de l'URSS (décisive dans le choix fait par le Daghestan, au tournant des années 1990, de rester dans la Fédération de Russie). 
3 L'un des aspects saillants de ce travail d'ensemble est la mise en lumière, au Daghestan, d'un clivage profond entre montagnards et gens des plaines, hérité des projets massifs de développement des piémonts de la période soviétique (rivalités entre montagnards avars et piémontais kumyks; conflits entre les Aki Tchétchènes et les Laks qui ont pris leur place après leur déportation en 1944). M. Gammer s'est également beaucoup interrogé, parfois de manière elliptique, sur l'impact qu'a pu avoir, à terme, l'installation en 1958 des Aki Tchétchènes privés de retour dans les bourgs de Hasav Jurt, Kizil Jurt et Buinaksk, sur l'apparition dans ces localités d'une forte politisation de l'islam. À noter enfin, contrastant avec la plupart des approches, une sensibilité aux divisions internes et changements de cap du personnel religieux de l'islam dans le Caucase Nord ${ }^{3}$.

Le présent volume, l'un des plus complets réalisés à ce jour sur la problématique de l'islam et du nationalisme dans le Caucase Nord à l'époque actuelle, poursuit et enrichit ces questionnements, avec un ensemble de contributions sur les processus politiques des années 1990 et 2000. Plusieurs articles de poids font le jour sur l'islamisation rapide de mouvements revendicatifs à l'origine purement ethniques et laïcs, même dans des régions réputées jusque-là superficiellement islamisées comme la république des Adygués ou celle des Kabardes et Balkars. Certains jettent un éclairage cru non seulement sur l'incapacité des États à prendre en compte ces phénomènes, mais aussi sur l'impact paradoxal qu'ont eu les politiques répressives mises en œuvre par la Russie dans l'ensemble du Caucase Nord sur la politisation accrue de l'islam dans l'ensemble de la région.

Une première mise au point, assez abstraite et nourrie de sources secondaires, sur le mode de mobilisation politique dans le Caucase Nord, insiste sur le primat des réseaux informels au sein des élites politiques et économiques. L'auteur y explique que la plupart des conflits ethniques de la région résultent, en fait, de rivalités interpersonnelles propres aux élites régionales, sans toutefois proposer de matériaux très convaincants (Maxim U. Barbashin, « Informal Power Structures in Russia and Ethno-Political Conflict in the Northern Caucasus ", p. 1-18). Une seconde étude assez généraliste traite de la pratique juridique musulmane dans le Caucase du Nord-Ouest au début de la période soviétique et de nos jours (Irina L. Babich, "Islam and the Legal System in the Northwestern Caucasus », p. 19-27). L'auteur y déroule une vision substantialiste de l'islam, assez commune dans l'ethnographie et l'orientalisme soviétiques, en affirmant, d'une part, que la pratique juridique des tribunaux souvent dits « de cadis " (kazijskie) laissés en place par les bolcheviks pendant les années 1920, était basée sur la « charia », et en reprochant d'autre part à l'islam contemporain son caractère essentiellement coutumier et «populaire». Si, dans le premier cas, l'auteur oublie le caractère essentiellement coutumier de la pratique juridique musulmane dans le Caucase jusqu'au début $\mathrm{du} \mathrm{xx}^{\mathrm{e}}$ siècle (les tribunaux islamiques servant par ailleurs de relais à l'autorité soviétique), dans le second, cette pratique s'instaure quasiment en autorité religieuse pour condamner les « déviations » de l'islam populaire par rapport à la norme littérale attitude courante chez les experts de l'ex-URSS et qui les rapproche beaucoup des Frères musulmans qui se nourrissent en partie de leurs travaux. Ces modes d'explication, curieusement, ne sont pas repris par les autres contributions du volume, dont la plupart préfèrent prendre comme base de réflexion des communautés - locales, régionales, ethniques -, des entités démographiques ou des mouvements politiques bien identifiés.

6 C'est le cas, en particulier, d'une première étude sur le thème du recueil, à la lumière du cas des Adygués (Chen Bram, "Re-Islamisation" and Ethno-Nationalism: The 
Circassians (Adyghe) of the Northwestern Caucasus and Their Diaspora", p. 28-49). L'auteur de ce travail étudie l'influence de la diaspora circassienne du Proche-Orient et de Turquie sur l'évolution du mouvement national depuis le milieu des années 1990, en insistant sur le rôle de cette dernière, mais aussi de missionnaires saoudiens et égyptiens, dans l'islamisation progressive du discours national en Adygué, notamment par l'ouverture - récemment combattue par les autorités régionales - d'écoles religieuses musulmanes. Chen Bram fait notamment le point sur l'impact exercé localement par le début de la seconde guerre de Tchétchénie à l'automne 1999 sur le développement rapide, au tournant du siècle, de groupes islamistes militants dans la région. La république des Adygués fait également l'objet d'une réflexion sur la perception par les citoyens ordinaires de notions telles que la liberté religieuse ou de parole et l'égalité des chances entre représentants des divers groupes ethniques qui composent sa population (Cemre Erciyes, « The Republic of Adygheia : Perceptions of Rights, Freedoms and Life Chances of Ordinary People », p. 50-67). L'auteur conclut de son enquête que les Adygués se voient avant toute chose comme citoyens de la Fédération de Russie, dont beaucoup continuent d'attendre - "par inertie soviétique », hasarde l'auteur - une meilleure redistribution des richesses de l'économie de marché. L'article suivant examine les relations mutuelles entre Balkars turcs et Kabardes circassiens, dans leur république commune de KabardoBalkarie, à travers la tentative de séparatisme des premiers en novembre 1996. Cette tentative fut alors avortée par le gouvernement local, dominé par les Kabardes, en échange de promesses de développement des régions montagneuses de peuplement balkar majoritaire (Julietta Meskhidze, "The Events of November 1996 in KabardinoBalkaria and Their Prehistory ", p. 68-87). Les évolutions plus récentes au sein de cette république réputée peu islamisée sont analysées à travers les attaques contre la capitale républicaine $\mathrm{Nal}^{\prime}$ čik, en 2003 et 2005, par des militants kabardes révoltés contre l'islamophobie des autorités et la politique de répression de toutes les pratiques religieuses jugées "non traditionnelles", une politique qui affecte particulièrement la jeunesse musulmane (Walter Richmond, « Russian Policies towards Islamic Extremism in the Northern Caucasus and Destabilization in Kabardino-Balkaria », p. 86-101).

7 La Tchétchénie fait l'objet d'un sous-ensemble très cohérent de quatre articles ouvert par une réflexion de Djohar Dudaev sur le recours aux "mémoires de conflits" pour la mobilisation en faveur de l'indépendance tchétchène en 1991-1996. L'auteur note l'islamisation des discours au sein du mouvement national tchétchène sous Mashadov et par la suite, en particulier au sein d'une jeunesse mue par un fort désir de revanche. Ici aussi l'auteur insiste sur le caractère totalement contre-productif de l'attitude des autorités russes confondant nationalisme, islamisme et extrémisme (Ekaterina Sokirianskaia, «Ideology and Conflict : Chechen Political Nationalism prior, and during, Ten Years of War », p. 102-138). La confusion des affiliations communautaires et des positionnements politiques à l'issue de deux décennies de conflit apparaît particulièrement dans l'aporie à laquelle se trouve confrontée l'ethnie Orstkhoy ou Karabulak du district de Sunja (revendiqué à la fois par la Tchétchénie et l'Ingouchie), entre une revendication d'irréductible spécificité et l'invocation récurrente d'une identité Vaïnakh transfrontalière, commune aux deux républiques nord-caucasiennes (Victor A.Shnirelman, "The Orstkhoy Revival: Identity and Border Dispute in the Northern Caucasus », p. 139-47). L'étude consacrée à la stratégie militaire développée par Aslan Mashadov à partir de 1999 (Yagil Henkin, «"I Can Fight, Army against Army" : The 1994-1996 Russo-Chechen War, Strategies and Misconceptions », p. 148-55) insiste sur la volonté du militaire indépendantiste de déployer, en 1994-1996, la stratégie militaire 
d'un État en guerre, axée notamment sur l'occupation de vastes régions par une armée conventionnelle, en rupture avec la tactique d'attaques contre des cibles civiles bientôt déployée par un Šamil' Basaev. La deuxième guerre de Tchétchénie est mentionnée, elle, principalement par le rôle qu'y ont joué les combattants étrangers et al-Qaida (Brian Glyn Williams, "Allah's Foot Soldiers: An Assessment of the Role of Foreign Fighters and al-Qa'ida in the Chechen Insurgency », p. 156-78).

8 Le Daghestan est évoqué par un rapide survol de l'essor de l'islam politique, à travers notamment la communauté rurale autonome de Karamahi et son anéantissement par la campagne militaire russo-daghestanaise de 1999, au lendemain de l'intervention armée des radicaux tchétchènes emmenés par Basaev et Khattab (Moshe Gammer, « From the Challenge of Nationalism to the Challenge of Islam : The Case of Daghestan », p. 179-93). Dans le sillage de ses précédents travaux, l'auteur et éditeur du volume prévient ses lecteurs contre l'utilisation par le pouvoir russe et l'administration daghestanaise du terme "wahhabite " à propos de toute forme de militance musulmane - et jette une lumière crue sur le rôle joué par la très officielle Direction spirituelle des musulmans du Daghestan dans l'islamisation des discours publics au sein de cette république multiethnique du Caucase, où la charge de mufti est traditionnellement l'objet de rivalités durables entre groupes ethniques dominants. Une rapide excursion dans le Caucase méridional nous conduit dans deux mosquées anticonformistes de Bakou, l'Abu Bakr sunnite et la Mosquée du Vendredi chiite qui, toutes deux, disposent d'une large audience dans la jeunesse et l'intelligentsia azerbaïdjanaise du fait du piétisme de la première - qui ne l'empêche pas d'être qualifiée de " wahhabite " par les autorités - et de la défense par la seconde de la démocratie occidentale, en rupture avec le conformisme politique des institutions religieuses musulmanes traditionnelles (Sofie Bedford, " "Wahhabis", Democrats and Everything in between: The Development of Islamic Activism in Post-Soviet Azerbaijan», p. 194-211). Une ultime contribution passe en revue les différentes appellations utilisées par les recensements pour les populations juives du Daghestan (Mark Tolts, «Demography of North Caucasian Jewry : A Note on Population Dynamics and Shifting Identities », p. 212-224).

9 Au total, on déplorera peut-être les jugements de valeur et les assertions à l'emportepièce qui caractérisent certaines études de ce recueil, ainsi qu'une tendance d'autres auteurs à ne pas dépasser dans leurs études le niveau de la simple chronique rétrospective. Il n'empêche: par son ampleur critique, cette collection d'articles témoigne d'une ambition de renouveau et de dépassement des catégories toutes faites de l'officialité, dans nos approches de l'islam politique dans le Caucase Nord pendant deux décennies d'une histoire particulièrement tourmentée. Une posture tournée vers l'innovation, et qui propose de nombreuses pistes de réflexion et de recherche aux spécialistes et autres "experts» de l'islam dans l'ex-domaine soviétique, dont la responsabilité dans les développements actuels est aussi plus qu'esquissée. 


\section{NOTES}

1. Voir récemment : Moshe Gammer, David J. Wasserstein, éds., Daghestan and the World of Islam, Helsinki : Suomalainen Tiedeakatemia (Annales Academiae Scientiarum Fennicae, Humanoria, 330), 2006.

2. Voir en particulier M. Gammer, "The Road Not Taken: Daghestan and Chechen Independence ", Central Asian Survey 24 (2), 2005, p. 87-108; id., "Between Mecca and Moscow : Islam, Politics and Political Islam in Chechnya and Daghestan ", Middle Eastern Studies 41 (6), 2005, p. 833-848, ainsi que mes comptes rendus de ces deux textes dans Central Eurasian Reader 2, 2010, p. 551-553.

3. Voir les considérations historiques sur les changements de stratégie vis-à-vis de l'occupant russe des voies mystiques Naqshbandiyya-Halidiyya et Qadiriyya-Kunta Hajji, tantôt du côté de l'opposition armée à la Russie, tantôt du côté de l'accommodation. 